\title{
Doppler Domain Decomposition of the Underwater Acoustic Channel Response
}

\author{
Salman Ijaz, António J. Silva, Orlando C. Rodríguez and Sérgio M. Jesus \\ Instituto de Sistemas e Robótica, Campus de Gambelas, \\ Universidade do Algarve, \\ 8005-139, Faro, Portugal \\ Email: \{ssiddiqui,asilva,orodrig,sjesus $\} @$ ualg.pt
}

\begin{abstract}
Most underwater applications are very sensitive to environmental perturbations like source/receiver motion and surface variations. In real conditions, the transmitted signal reaches the receiver through different paths where each path is affected by different environmental perturbations. Due to the interaction with these environmental variations different Doppler is induced in each path. By observing the Doppler for the whole signal it is not possible to determine the contribution of each environmental variation. The main goal of this paper is to isolate different paths and analyse the effects of the environmental variations on each path in terms of the induced Doppler. A new technique called Time Windowed Doppler Spectrum is proposed which has been found very effective in tracking the Doppler due to each path separately. By using this technique it can be shown that it is possible to distinguish between surface reflected and direct path by observing the Doppler variations associated with each path. The surface induced Doppler was observed using this technique by analysing the temporal evolution of the surface reflected path. The surface variability effects the Doppler in terms of stretching and shortening the path between the transmitter and receiver. The data processed in this paper was acquired during CALCOMM'10 Experiment which took place in June 2010 at the south coast of Portugal.
\end{abstract}

\section{INTRODUCTION}

Most underwater applications are greatly affected by source/receiver motion and surface variations. Due to these variations the underwater channel changes strongly with time. In order to achieve good performance it is important to design some techniques to track these changes. The main idea of this paper is to address this problem and propose a method to infer information about these perturbations based on Doppler analysis.

The underwater acoustic channel is characterized by a long multipath spread where each path is subject to distortion due to the motion of the transmitter and/or the receiver, and the sea surface. In recent literature many studies focus on the simulation of the sea surface variations and their effects on the Doppler spread spectrum. In [1] two simulation methods are described for modelling time varying sea surface using ray theory and ray based formulation of the Helmholtz integral equation with a time domain Kirchoff approximation. In [2] a matched filtering technique is used to estimate Impulse Response (IR) and to study the effect of environmental variations caused by source/array movement and sea surface motion on the impulse response. In both [1] and [2] the Doppler shifted replicas of the transmitted signals are matched filtered with the received signal and depending on the peak in the ambiguity plane source/receiver motion and surface variations are approximated.

Due to source and/or receiver motion and the surface variations each path is affected by different dynamics of the environment. To study the effect on each path, Time Windowed Doppler Spectrum (TWDS) is used. TWDS is computed by windowing the impulse response over time. Computing TWDS for different time instants revealed that the Doppler spectrum is changing with time. This change is expected to be due to the surface waves and source and/or array motion. In this paper the TWDS technique is applied to real data acquired on a single hydrophone and variations in Doppler are clearly found. A time-frequency based technique similar to the one proposed in [3], is used to improve the resolution of the results and to see the temporal evolution of Doppler due to surface reflected arrival over the transmission time. A very interesting behaviour is observed in surface induced Doppler due to the relative motion of the source, receiver and the surface which led to the extension of this analysis to other hydrophones in the array.

The real data of CALCOMM'10 sea trial is presented in this paper. CALCOMM'10 took place at the south coast of Portugal from $22^{\text {nd }}$ to $24^{\text {th }}$ of June 2010 . The data set presented in the paper has duration of $15 \mathrm{sec}$ and contains chirp signals with the central frequency of $3.125 \mathrm{KHz}$, duration of $0.1 \mathrm{sec}$ and a repetition rate of $0.3 \mathrm{sec}$. The same scenario is also modelled using bellhop [4]. By computing the ray tracing diagram it is observed that the first arrival is the combination of the direct path and surface reflected path, reaching the hydrophone at the same time which results in destructive interference at the reciever.

The paper is organized as follows: Section II elaborates the theoretical background and mathematical modelling of the problem. Section III gives the description about the CALCOMM10 sea trial. Section IV explains modelling results with bellhop and some preliminary results. Section V presents the data processing and results with the real data and Section VI presents the conclusions and future work.

\section{THEORATICAL BACKGROUND}

In underwater transmission systems, the transmitted signal reaches the hydrophone through different paths which can 
be categorized as the surface reflected, bottom reflected and water column refracted paths. The water column refracted paths are mostly direct paths which are affected by the sound speed profile which is a function of depth and range. Each of these paths has different sensitivities to the environmental variations. The water column refracted paths are only sensitive to the source and array motion while the surface reflected path is also affected by the surface motion in addition to the source and array motion.

The geometric variations like source/array movements produces compression/expansion in the transmitted signal which induces different Doppler corresponding to each path. This can be analysed at the path level in terms of time-variable IR $h_{m p}$ for a single propagation path $p$ in [5]

$$
h_{m p}(t, \mu)=g_{m p}\left(\mu+(t-\mu) \frac{v}{c}\right) e^{j \omega_{c}\left(\mu+(t-\mu) \frac{v}{c}\right)}
$$

where $g_{m p}(t, \mu)$ is a single path, $p$, propagating between the source and the hydrophone, $m$, transmitted at an instant $t=0$ and received at the hydrophone after a delay $\mu$. Due to the channel properties variability the length, $l_{p}(t)$ of the path changes with a velocity $v=\partial l_{p}(t) / \partial t$. The ratio between such velocity and the sound speed, $c$, induces a delay variation in the $g_{m p}(t, \mu)$ argument and a frequency shift given in (1) by the complex exponential. Such frequency shift is responsible for the Doppler spread that also depends on the central frequency, $\omega_{c}$, of the narrowband transmitted signal. Equation (1) gives the time variable IR for a single path which can be generalized to $p$ paths by doing a weighted sum of all the delayed replicas of the transmitted signal.

$$
h_{m}(t, \mu)=\sum_{p} h_{m p} \delta\left(\mu-\mu_{m p}\right)
$$

where $h_{m}(t, \mu)$ incorporates all the Doppler experienced by the $m^{\text {th }}$ hydrophone of the array due to all arriving paths. To compute the Doppler corresponding to each hydrophone Fourier transform is taken with respect to time in equation (2) which gives the spreading function $H_{m}(\phi, \mu)$ as

$$
H_{m}(\phi, \mu)=\int_{-\infty}^{\infty} h_{m}(t, \mu) e^{j 2 \pi \phi t} d t
$$

where $\phi$ is the Doppler induced due to all arriving paths at hydrophone $m$.

Figure 1 shows the simplified ray diagram showing two paths $p_{1}$ and $p_{2}$ from the source $T$ to the hydrophone $R$. Path $p_{1}$ is the direct path from the source to the receiver while $p_{2}$ is the surface reflected path. Considering only the surface induced motion, path $p_{1}$ is only affected by the up-down and range movement of the surface suspended array while $p_{2}$ is directly affected by the surface motion as well as the array motion. In figure $1, V_{T}, V_{R}$ and $V_{S}$ are the constant velocity vectors at the transmitting, receiving and the surface reflection point respectively. $\hat{n}_{T}^{\prime}$ and $\hat{n}_{R}^{\prime}$ are the unit vectors in the directions of the propagation of the transmitted and received signal for
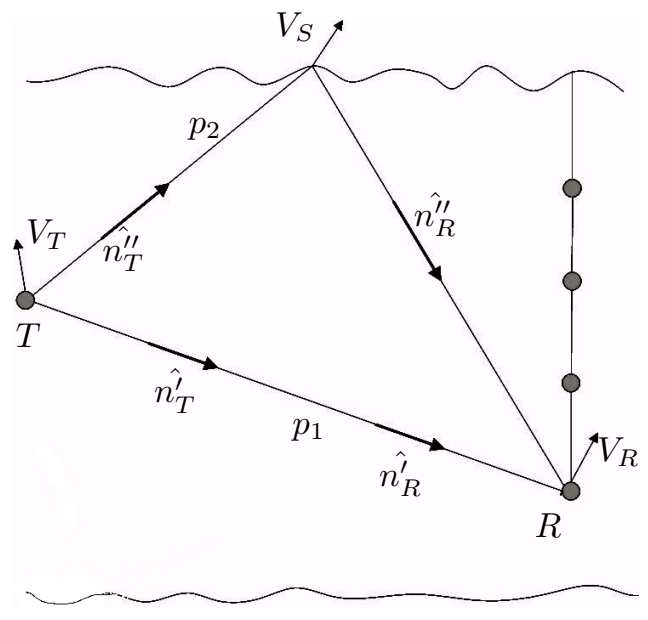

Fig. 1. Two arrivals from Transmitter to Receiver and their velocity projections

the direct path while $\hat{n}_{T}^{\prime \prime}$ and $\hat{n}_{R}^{\prime \prime}$ are the unit vectors for the surface reflected path.

As explained before the change in path length is a function of velocity so the Doppler is induced in both paths by the source motion and the surface motion which is given by [6]

$$
\phi_{A}=-(1-s) f_{c}
$$

where $\phi_{A}$ is the actual Doppler shift in hertz and $s$ is the time compression/expansion factor given by

$$
s=\frac{\left(1-V_{S} \cdot \hat{n}_{T} / c\right)\left(1-V_{R} \cdot \hat{n}_{R} / c\right)}{\left.\left(1-V_{S} \cdot \hat{n}_{R} / c\right)\right)\left(1-V_{T} \cdot \hat{n}_{T} / c\right)}
$$

For path $p_{1}$ the compression/expansion factor $s^{\prime}$ will be given by

$$
s^{\prime}=\frac{\left(1-V_{R} \cdot \hat{n}_{R}^{\prime} / c\right)}{\left(1-V_{T} \cdot \hat{n}_{T}^{\prime} / c\right)}
$$

Substituting this value in equation (4) and substituting the value of $s^{\prime}$ in equation (2) the actual Doppler $\phi_{A}^{\prime}$ becomes

$$
\phi_{A}^{\prime}=\frac{\left(V_{T} \cdot \hat{n}_{T}^{\prime}-V_{R} \cdot \hat{n}_{R}^{\prime}\right) / c}{1-V_{T} \cdot \hat{n}_{T}^{\prime} / c} f_{c}
$$

Equation (7) relates the actual Doppler shift $\phi_{A}$ with the relative velocities of the source and the hydrophone. Considering a static hydrophone, when the source is moving away from the hydrophone then $\hat{n}_{T}^{\prime}=-\hat{n}_{R}^{\prime}$ and $\phi_{A}^{\prime} \leq 0$. When the source moves towards the hydrophone one can get that $\hat{n}_{T}^{\prime}=\hat{n}_{R}^{\prime}$, and $\phi_{A}^{\prime} \geq 0$.

Solving (4) for path $p_{2}$ one can get that

$$
\phi_{A}^{\prime \prime}=\frac{\left(\left(V_{T}-V_{S}\right) \cdot \hat{n}_{T}^{\prime \prime}-\left(V_{S}-V_{R}\right) \cdot \hat{n}_{R}^{\prime \prime}\right) / c}{\left(1-V_{S} \cdot \hat{n}_{R}^{\prime \prime} / c\right)\left(1-V_{T} \cdot \hat{n}_{T}^{\prime \prime} / c\right)} f_{c}
$$

where $\left(V_{T}-V_{S}\right)$ and $\left(V_{S}-V_{R}\right)$ are the source and the hydrophone velocities relative to the surface velocity $V_{S}$. So equation (8) shows the effect of the surface variations on Doppler shift $\phi_{A}^{\prime \prime}$ for the surface reflected path. Similar 
analysis can be made in terms of the Doppler shift and the relative motion of the source and the hydrophone. Considering a static hydrophone, when the source is moving away from the hydrophone then $\hat{n}_{T}^{\prime \prime}=-\hat{n}_{R}^{\prime \prime}$ and $\phi_{A}^{\prime \prime} \leq 0$. When the source moves towards the hydrophone one can get that $\hat{n}_{T}^{\prime \prime}=\hat{n}_{R}^{\prime \prime}$, and $\phi_{A}^{\prime \prime} \geq 0$.

\section{EXPERIMENT DESCRIPTION}

The data analysed in this paper was collected during the CALCOMM'10 experiment, which took place off the south coast of Portugal, about 12 nautical miles south east of Vilamoura, from $22^{\text {nd }}$ to $24^{\text {th }}$ of June 2010. The main objectives of the experiment were to collect field calibration data for tomography purposes and transmit communication signals in different frequency bands to analyse the performance of underwater communication systems.

The acquisition system used for gathering the data comprised of two acoustic oceanographic buoys (AOBs), one with 8 hydrophones and the other with 16 hydrophones [7].

The data presented in this paper was taken on Day 2 of the experiment from the 16 hydrophone array in which all the hydrophones are equally spaced at $4 \mathrm{~m}$. The results presented in this paper were acquired from the communication signals which comprises LFMs and QPSK modulated signals. Some details about the different transmitted signals during the experiment are shown in table 1.

Figure 2 (a) shows a downward refracting sound speed profile measured during the experiment. Figure 2 (b) shows the source and receiver locations during data transmissions plotted over the bathymetric map of the area where the experiment took place. During the data transmission analysed in this paper, the source array distance was around $850 \mathrm{~m}$ with a downslope bathymetry, the source was located at about $12 \mathrm{~m}$ depth and the first hydrophone of the array was located $6.3 \mathrm{~m}$ from the surface.

\section{Preliminary Observations}

The discussion of this section is mainly focused on the low frequency chirps (LF_AERU1 code, see table 1) with the central frequency of $3.125 \mathrm{KHz}$. The received chirps were first filtered by a band pass filter to remove out of band noise, then the filtered signal was converted to baseband and pulse

TABLE I

SIGNAL SPECIFICATION FOR THE CALCOMM'10 EXPERIMENT

\begin{tabular}{|c|c|c|c|c|}
\hline Code & Type & $\begin{array}{c}\text { Baud Rate } \\
(\mathrm{bps})\end{array}$ & $\begin{array}{c}\text { Start-stop freq } \\
(\mathrm{KHz})\end{array}$ & $\begin{array}{c}\text { Duration } \\
(\mathrm{sec})\end{array}$ \\
\hline LF_AERU1 & LFM & - & $2.64-3.75$ & 0.1 \\
\hline MF_AERU1 & LFM & - & $5.0-7.0$ & 0.1 \\
\hline MF_PASU1 & LFM & - & $5.0-7.0$ & 0.1 \\
\hline HF_PASU1 & LFM & - & $10.0-15.0$ & 0.1 \\
\hline LF_AERU2 & QPSK & 500 & $2.9-3.5$ & 30.2 \\
\hline MF_AERU2 & QPSK & 1000 & $5.5-7.0$ & 30.2 \\
\hline MF_PASU2 & QPSK & 1000 & $5.5-7.0$ & 30.2 \\
\hline HF_PASU2 & QPSK & 2000 & $11.0-14.0$ & 30.2 \\
\hline
\end{tabular}

(a)

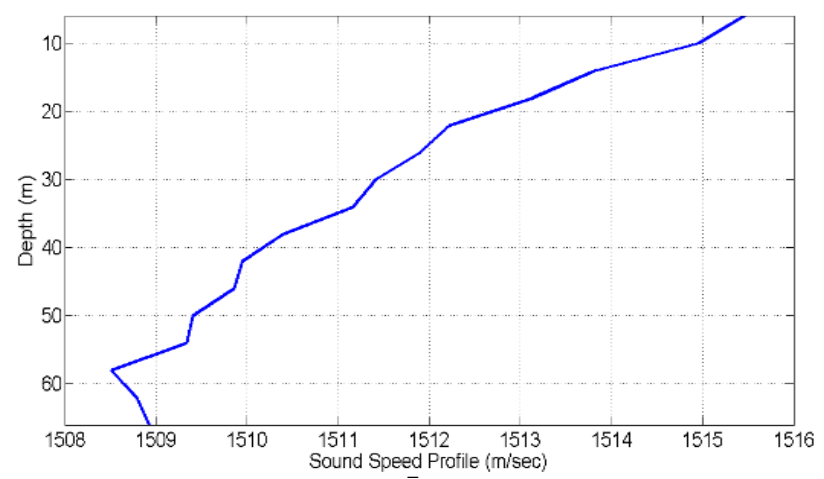

b)

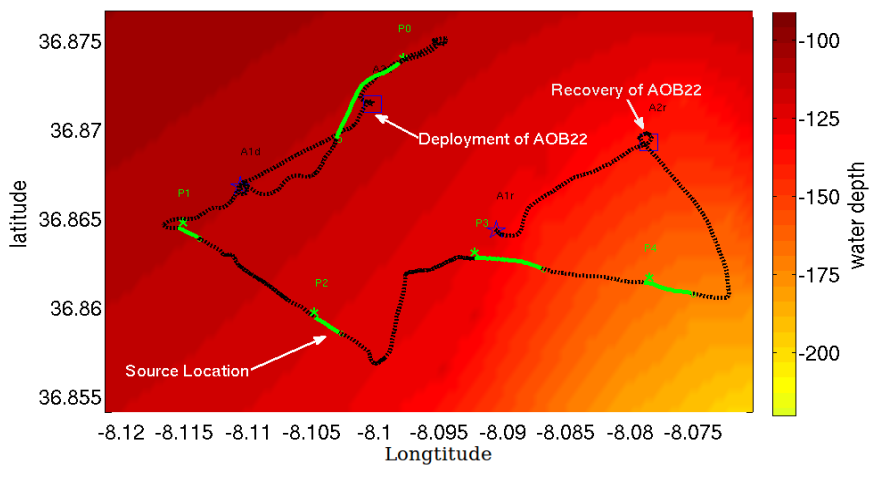

Fig. 2. (a) Downward Refracting sound speed profile during Day 2 (b) Day 2 bathymetry map of the work area with GPS estimated locatons of AOB21 and AOB22 deployments and their recovery, ship/source track (dotted lines) and ship track during communication events (green lines)

(a)

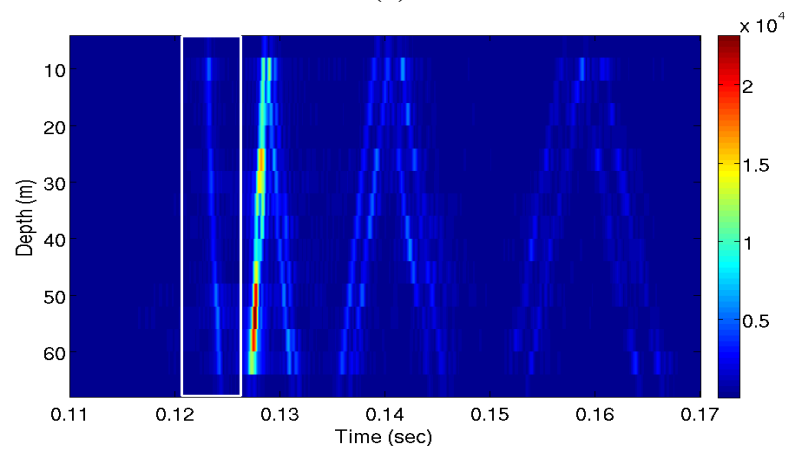

(b)

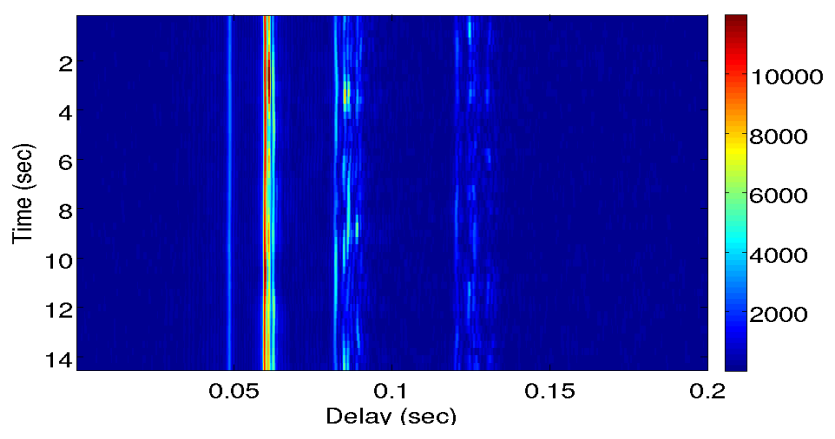

Fig. 3. Impulse Response Estimates: (a) for 16 hydrophone array and white lines showing the selected wavefront (b) the temporal evolution of IR along $15 \mathrm{sec}$ of transmission for channel 3 
compressed with the transmitted chirps to get the channel IR estimate. Figure 3 (a) shows the estimated arriving pattern that comprises an impulse reaching the array of hydrophones through different paths and (b) shows the temporal evolution of IR along the $15 \mathrm{sec}$ of transmission at hydrophone 3 .

The arriving pattern in figure 3 (a) shows the impulse reaching the array at different delays. Two abnormaltities are observed in this scenario. Firstly it is expected to see a strong (high energy) arrival which can be due to direct path between the source and the array but this is not the case in this scenario. The initial wavefront has lesser energy as compared to the second wavefront. Secondly the second high energy wavefront has a positive slope so the signal is reaching the bottom hydrophone first which suggests that this is a bottom reflected wavefront. The source is located very near to the surface so the surface reflected wavefront should reach the hydrophones before the bottom reflected wavefront. The reason for these abnormalities can be explained by modelling the environment with Bellhop.

Figure 4 shows the ray tracing diagram of the enviroment. By analysing the path delay given by Bellhop it can be seen that the direct arrivals (red lines) and the surface reflected arrivals (black lines) are reaching the hydrophone at the same time so the first wavefront is the superposition of the direct and the surface reflected arrivals. Due to the reflection from the surface the phase of the surface reflected arrival changes which results in a destructive interference of the surface reflected arrival and the direct arrival resulting that they can not be distinguished in figure 3 .

\section{DAta Processing And Results}

The proposed Doppler analysis method assumes that each arrival is affected by different dynamics of the environment. The direct arrival is affected by the source and the hydrophone motion while the surface reflected arrival is affected by source motion, hydrophone motion and the surface motion. The main objective of this method is to study the effects of the environmental dynamics on each arrival. Figure 5 (a) shows the zoomed version of the first path in figure 3 (a) which corresponds to a delay of $0.124 \mathrm{sec}$. In this figure it is not possible to extract any information regarding the number of the arrivals . Figure 5 (b) shows the corresponding Doppler spread of the first wavefront. Three lobes are clearly visible in the figure. The middle lobe at $\sim-0.01 \mathrm{~Hz}$ is expected to be due to the main arrival. Some ambiguity persists regarding the other two side lobes at $0.1 \mathrm{~Hz}$ and $-0.2 \mathrm{~Hz}$ as they may be due to two different arrivals or a single arrival which is affected by different dynamics of the environment. If the two side lobes are due to a single arrival then the two lobes should not appear simultaneously in a short time slot.

Time Windowed Doppler Spectrum (TWDS) is used to study the temporal evolution of the selected wavefront. TWDS is computed by windowing the IR estimate, shown in figure 5 (a), along time and computing the Doppler for the corresponding window of the IR. Root Raised Cosine window is used for windowing in this analysis because of its efficiency in reducing the side lobes. TWDS for an input signal $x(t)$ can be shown as the short time fourier transform of a signal [8]

$$
S(t, \omega)=\int_{-\infty}^{-\infty} x(t) w(t-\tau) e^{-j \omega \tau} d \tau
$$

where $w(t)$ is the window function which is root raised cosine function in this case.

Figure 6, 7 and 8 (a) show the TWDS results computed with a window of $4 \mathrm{sec}$ where the window slides $0.5 \mathrm{sec}$ from 6 to 7 and to 8. Figure 6, 7, 8 (b) show the corrensponding Doppler spread summation along the delay axis. The following

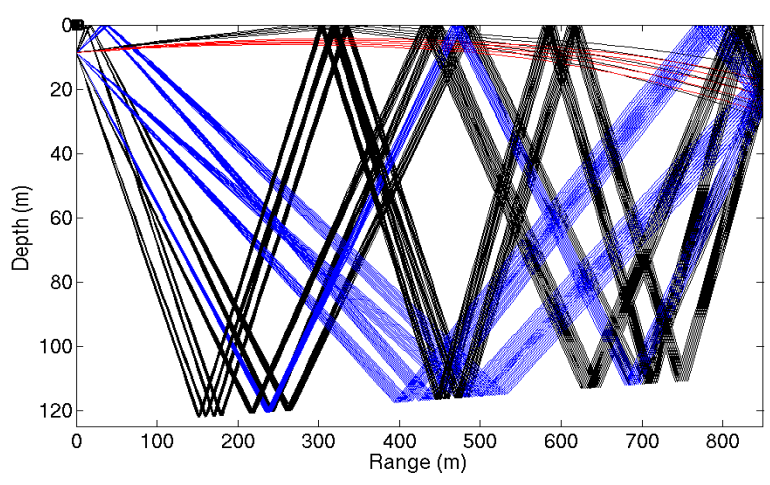

Fig. 4. Ray Tracing Diagram of the environment

(a)

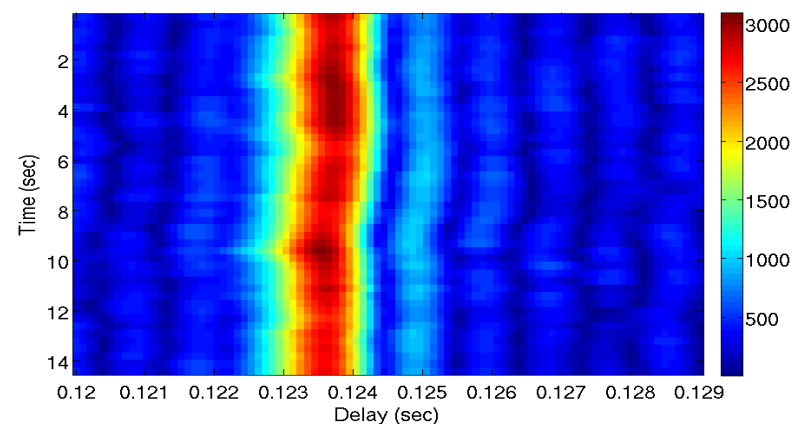

(b)

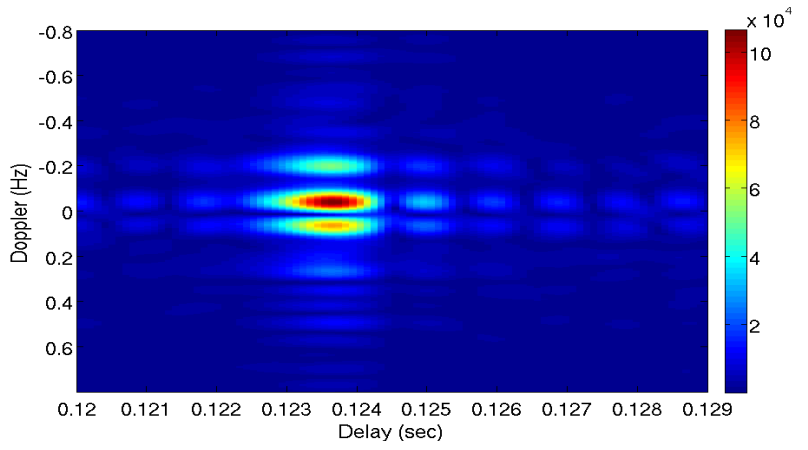

Fig. 5. (a) Variability of the selected wavefront shown in figure 3 (a), (b) Corresponding Doopler spread of the selected wavefront 
(a)

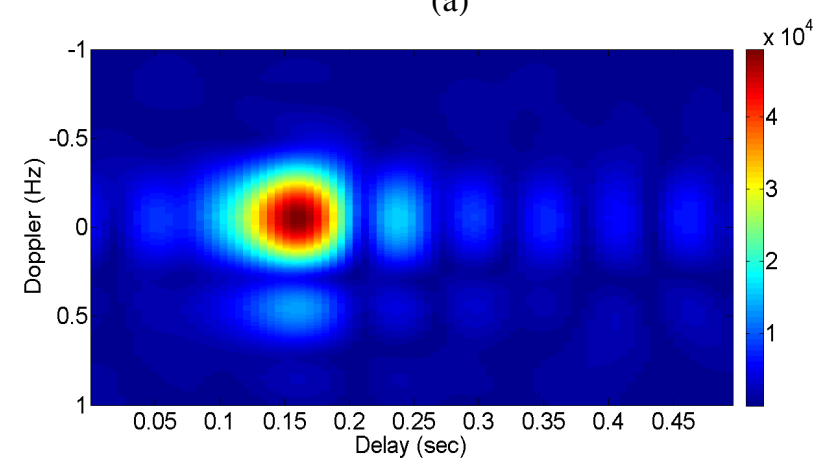

(b)

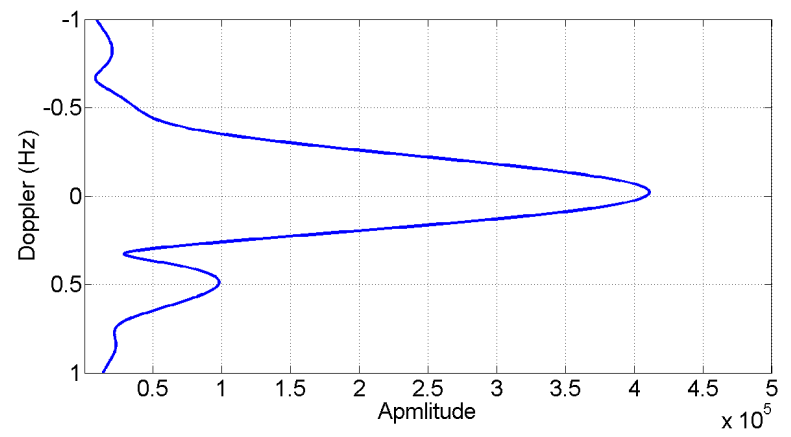

Fig. 6. (a) Doppler computed only for $4 \mathrm{sec}$ time window where two lobes can be seen i) due to one main arrival at $\sim 0.01 \mathrm{~Hz}$ and ii) due to surface reflected arrival at approximately $0.5 \mathrm{~Hz}$ (b) Doppler summation along the delay axis

(a)

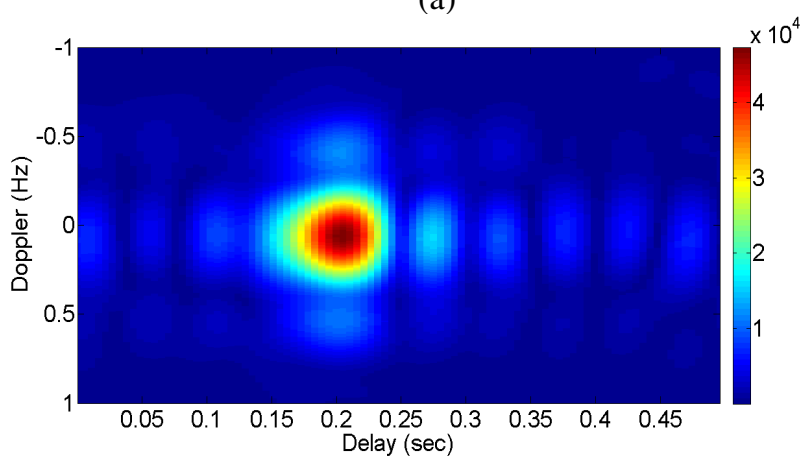

(b)

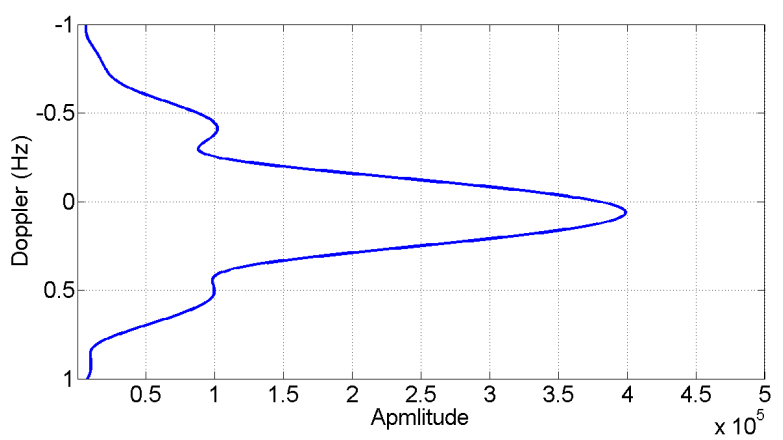

Fig. 7. (a) Doppler computed only for $4 \mathrm{sec}$ time window where three lobes can be seen i) due to one main arrival at $\sim 0.1 \mathrm{~Hz}$ and ii) due to surface reflected arrival at $\sim-0.4 \mathrm{~Hz}$ and $\sim 0.5 \mathrm{~Hz}$ (b) Doppler summation along the delay axis

(a)

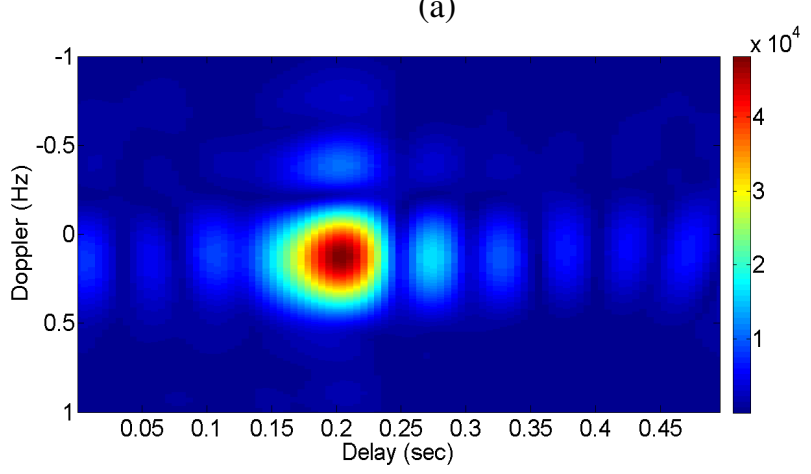

(b)

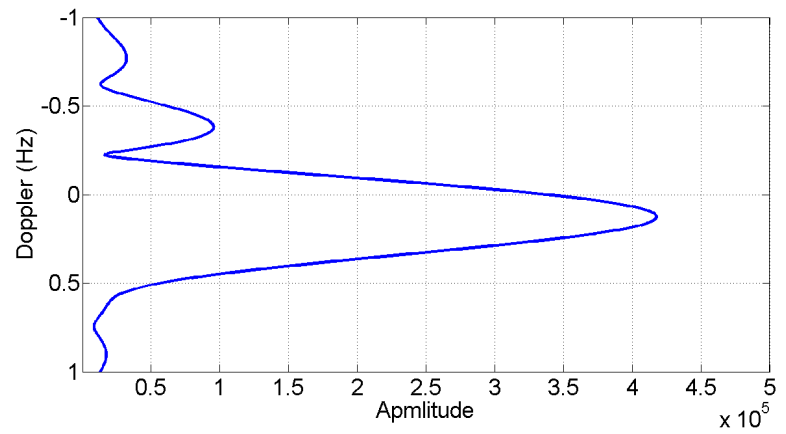

Fig. 8. (a) Doppler computed only for $4 \mathrm{sec}$ time window where two lobes can be seen i) due to one main arrival at $\sim 0.1 \mathrm{~Hz}$ and ii) due to surface reflected arrival at $\sim-0.4 \mathrm{~Hz}$ (b) Doppler summation along the delay axis 
observations can be deduced from these figures; (i) figure 6 (a) shows a main spot at $-0.01 \mathrm{~Hz}$ and a relevant side spot at $\sim 0.5 \mathrm{~Hz}$; (ii) figure 7 (a) shows a main spot at $0.1 \mathrm{~Hz}$ and two side lobes at approximately $-0.4 \mathrm{~Hz}$ and $0.5 \mathrm{~Hz}$, and (iii) figure 8 (a) shows the main spot at $0.1 \mathrm{~Hz}$ and the side spot at $0.4 \mathrm{~Hz}$. In all cases the main spot is expected to be due to the direct path ( $p_{1}$ in figure 1 ) which gives different Doppler values due to the array motion for the 3 different windows, nevertheless with the values around zero. The side spots are expected to be due to the surface motion that makes the surface reflected path ( $p_{2}$ in figure 1) larger and shorter, in the following manner : in case (i), the Doppler value is positive revealing that for that window the surface wave is moving up and the path is becoming larger; in case (iii) the Doppler value is negative revealing that for that window the wave is moving down and the path is becoming shorter; and for the case (ii) there are two side lobes one negative and the other positive revealing that for that time window the surface wave is crossing a crest, where the path is becoming larger before the crest and becoming shorter after the crest.

Figure 9 (a) shows the summation along the delay axis of the Doppler-delay diagram for the whole $15 \mathrm{sec}$ of transmission for hydrophone 3 . The main idea is to show the complete evolution of Doppler along time. Due to the windowing effect, the image becomes very blur, so it is difficult to identify the Doppler due to the surface motion. This blurring effect is due to the resolution trade-off which is one of the drawback of TWDS.

Resolution trade-off suggests that in order to get a good time resolution a narrow window in time domain $w(t)$, is required while for good Doppler domain resolution a narrow frequency domain window $W(\omega)$ is required in Doppler domain. But both $W(\omega)$ and $w(t)$ can not be optimized at the same time to get a good resolution in both time and Doppler domain. In order to improve the resolution of the image, Time Frequency (TF) analysis technique, proposed in [3] is used.

This technique helps in overcoming the resolution trade-off by using Wigner Transform (WT). In [3] the Wigner Transform for input signal $s(t)$ is given by

$$
S(t, \omega)=\int_{-\infty}^{-\infty} s^{*}\left(\frac{t-\tau}{2}\right) \cdot s\left(\frac{t+\tau}{2}\right) e^{-j \omega \tau} d \tau
$$

The Wigner transform gives crisp resolution due to the correlation operation. As proposed in [3], the resolution trade-off can be overcome by simply multipying TWDS with WT. Figure 9 (b) shows the results of this technique for hydrophone 3. By comparing the results in figure 9 (a) and (b) it can be observed that the Doppler due to direct arrival and the surface reflected arrival can easily be distinguished. The Doppler values starts from a negative value and two transitions occur at $\sim 4 \mathrm{sec}$ and $\sim 10 \mathrm{sec}$. Another interesting thing to observe is the variation in the Doppler due to the direct arrival. The direct arrival is only affected by the source and hydrophone motion so it is expected that this variation is due to the source motion but the source is suspended from the surface so the direct arrival (a)

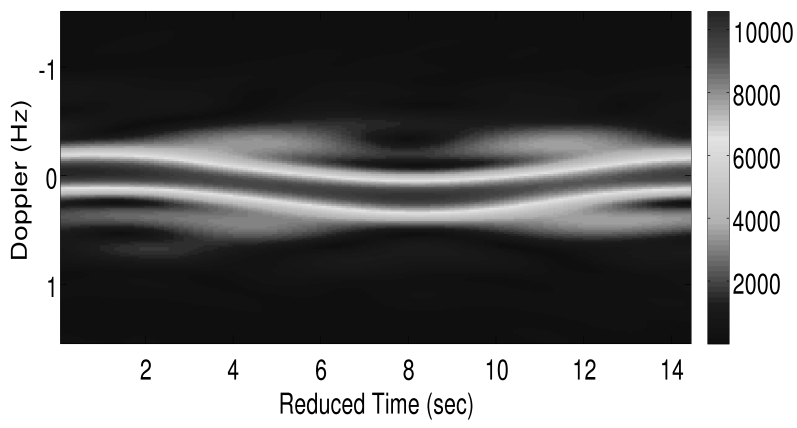

(b)

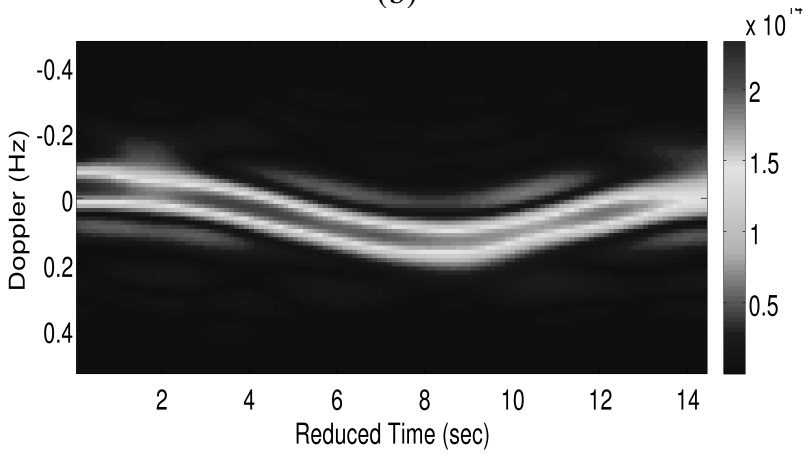

Fig. 9. (a) Summation along the delay axis of Doppler-delay diagram computed by only TWDS analysis (b) Summation along the delay axis of Doppler-delay diagram computed by combination of TWDS and WT

(a)

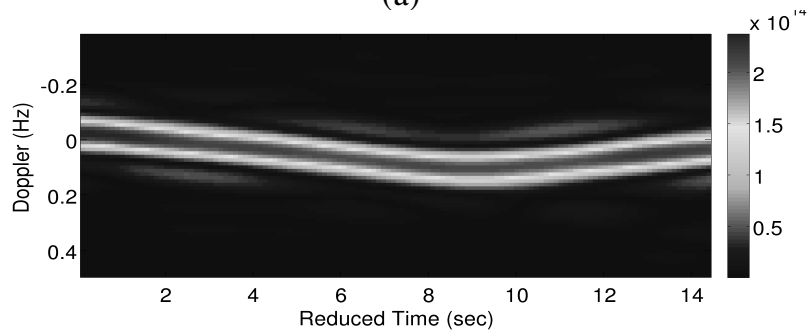

(b)

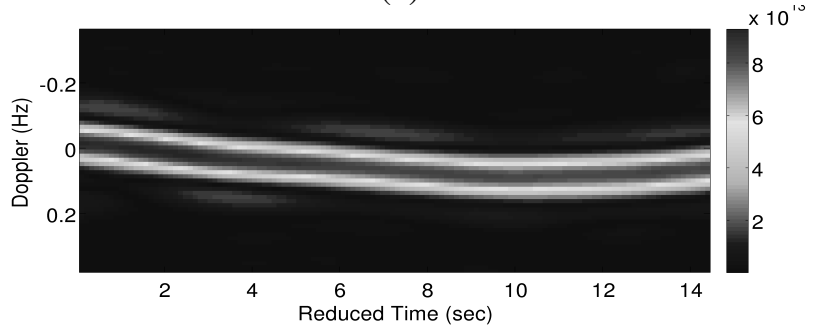

(c)

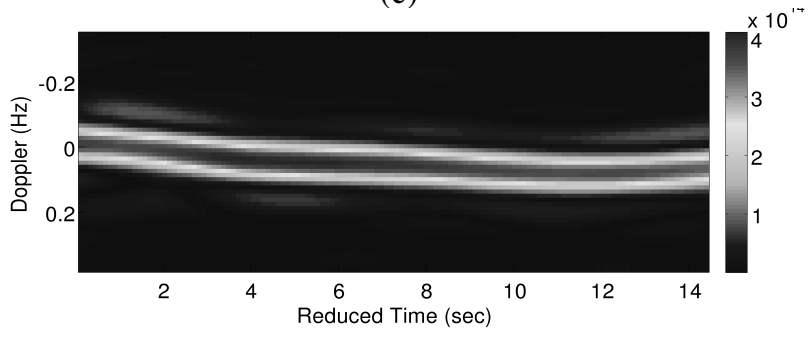

Fig. 10. Summation along the delay axis of Doppler-delay diagram computed by combination of TWDS and WT for (a) Hydrophone 4 at $18.3 \mathrm{~m}$ depth (b) Hydrophone 5 at $22.3 \mathrm{~m}$ depth (c) Hydrophone 6 at $26.3 \mathrm{~m}$ depth 
is also affected by the surface motion which can be seen in figure 9.

Figure 10 (a), (b), (c) show the results of TWDS-WT analysis for hydrophone 4,5 and 6 at $18.3 \mathrm{~m}, 22.3 \mathrm{~m}$ and $26.3 \mathrm{~m}$ respectively. In figure 10 (a), (b), (c) the Doppler due to direct path and surface reflected path can easily be distinguished. The strong Doppler in all three figures is due to the direct path between the source and the hydrophone. The other Doppler is expected to be due to a single surface reflected path because of the fact that its value is not positive and negative at the same time. Moreover the variations between positive and negative values depend on the contraction and expansion of the surface reflected path due to surface motion.

\section{CONCLUSion AND Future Work}

In this paper Doppler domain analysis of the underwater acoustic channel is done to analyse the time variability of the channel. In underwater multipath environment each path is affected by different environmental variations. The effects of these variations can be studied by observing the Doppler variations in the Impulse Response. These Doppler variations are observed due to contraction and expansion in the path length between the source and the receiver. Time Windowed Doppler Spectrum is used to study these effects on the channel impulse response. By isolating a single wavefront and observing its temporal evolution it is revealed that it comprises of two arrivals (direct and surface reflected) interfering destructively at the receiver. To study the effect of surface motion, the temporal evolution of the Doppler induced in the surface reflected path is studied in detail.

Future work involves computing the angles of departure and reception for direct path and surface reflected path between the source and the hydrophone. By doing so it can be possible to find the numerical value of the surface velocity using the proposed model. As explained before that the surface reflected path is not only affected by the surface motion but also by the source and array motion, so in order to find the surface velocity the projection of the source and array velocity is required along the surface reflected path. For computing these projections the departure and the reception angles are required.

\section{ACKNOWLEDGMENT}

This work was partially funded by FCT Portugal (ISR/IST plurianual funding) through the PIDDAC Program funds and project PHITOM (PTDC/EEATEL/71263/2006). The authors would like to thank project WEAM(PTDC/ENR/70452/2006) who are the partner of CALCOMM'10 Experiment, chief scientist Dr. Paulo Felisberto and the ship crew for their support during CALCOMM'10.

\section{REFERENCES}

[1] Martin Siderius, and Michael B. Porter,. Modeling broadband ocean acoustic transmissions with time-varying sea surfaces. J. Acoust. Soc. Am., 124:137-150, July 2008.

[2] Nicolas F. Josso, Jerome I. Mars, Cornel Ioana, Cedric Gervaise, and Yann Stephan. On the consideration of motion effects in the computation of impulse response for underwater acoustics inversion. J. Acoust. Soc. Am., 126:1739-1751, Oct. 2009.
[3] Soo-Chang Pei, and Jian-Jiun Ding. Relations between gabor transforms and fractional fourier transforms and their applications for signal processing. IEEE Transactions on Signal Processing., 55, no. 10:4839, Oct. 2007.

[4] Michael B. Porter, and Y. C. Liu. Finite-element ray tracing, theoretical and computational acoustics. World Scientic Publishing Co., 2, 1994.

[5] A. Silva, O. Rodriguez, F. Zabel, J. Huilery, and S. M. Jesus. Underwater acoustics simulations with time variable acoustics propagation model. Proceeding of 10th European Conference on Underwater Acoustics, 2:989-996, July. 2010.

[6] Lawrence. J. Ziomek. Fundamentals of Acoustic Field Theory and SpaceTime Signal Processing. CRC, Boca Raton. FL, 1995.

[7] A. Silva, F. Zabel, C. Martins,. Acoustic oceanographic buoy: a telemetry system that meets rapid environmental assessment requirements. Sea Technology, 47:15-20, Sept. 2006.

[8] Leon. Cohen. Time-Frequency Analysis. Prentice Hall NJ, 1995. 